\title{
The practice of water operation and groundwater protection in the middle route of South-to-North Water Transfer Project
}

\author{
Li Jia ${ }^{1}$, Gao Yuan-yuan ${ }^{1}$, Yan Liu-qing ${ }^{2}$, Yao Jian-wen ${ }^{1}$, Chen Gui-fang ${ }^{1}$ \\ ${ }^{1}$ Bureau of South to North Water Transfer of Planning, Designing and Management, Ministry of Water Resources, Beijing 100038, \\ China; ${ }^{2}$ China Institute of Water Resources and Hydropower Research, Beijing 100038, China
}

\begin{abstract}
The South-to-North Water Transfer Project plays an important role in guaranteeing the sustainable development of economy and society in North China. The first phase of the middle route of the South-toNorth Water Transfer Project (m-SNWTP) has been running safely in the past three years, and the dispatched water amount is increasing. This paper mainly describes the water operation of the m-SNWTP and the groundwater protection in the water-receiving areas. Up to the end of June 2018 , more than $1.4 \times 10^{10} \mathrm{~m}^{3}$ water had been supplied to the m-SNWTP benefited regions, including $8.65 \times 10^{8} \mathrm{~m}^{3}$ environmental water. In addition, the m-SNWTP provides water source guarantee for the environment restoration. Over $1.326 \times 10^{9}$ $\mathrm{m}^{3}$ overexploited groundwater has been reduced in the urban areas of the water-receiving areas, and the continuous decline of groundwater level has been effectively curbed.
\end{abstract}

\section{Introduction}

China is a country short of water resources. Although the total amount of water resources reaches about 2800 billion cubic meters, water resources per capita is only $1 / 4$ of the world average due to the large population in China. Meanwhile, water resource is unevenly distributed in both space and time, and it is incompatible with population, cultivated land and economic development ${ }^{[1]}$. Therefore, appropriate allocation of limited water resources is an important issue for sustainable development. According to the studies on this issue for several decades, the South-toNorth Water Transfer Project (SNWTP) that diverts water from the Yangtze River to the North China is proposed ${ }^{[2]}$.

The Yangtze River is the largest river in China with an average annual runoff of about 960 billion cubic meters. $94 \%$ of water from the Yangtze River flows into East China Sea, so it is possible to divert some water from the Yangtze River basin to alleviate the water shortage in North China. With 50 years survey, design and research, three diversion areas in the upper, middle and lower reaches of the Yangtze River have been designed. The three routes were proposed, namely the east, the middle and the west routes. The middle route was fully operated on December $12,2014^{[3-6]}$. This paper mainly describes the water dispatching situation since the operation of the middle route of the South-to-North Water Transfer Project $(\mathrm{m}-\mathrm{SNWTP})$ and the groundwater protection in the corresponding water-receiving areas (WRA).

\section{The water operation of $\mathrm{m}$-SNWTP}

The m-SNWTP gets water from Taocha intake of Danjiangkou reservoir and passing through four provinces and cities, namely Henan province, Hebei province, Beijing and Tianjin. The total length of the main water transportation is $1277 \mathrm{~km}$, and the canal to Tianjin is 155 $\mathrm{km}$ (Figure 1). The key of the scheme is to solve urban water short-ages of over 20 medium and large cities. Besides, the ecological and environment water and agricultural water uses along the line is also taken into $\operatorname{account}^{[7-8]}$.

In the planning of the m-SNWTP, the water supply of the middle and lower reaches of Danjiangkou reservoir and the WRA were adjusted, and it was concluded that the recent average adjustable water amount is $9.7 \times 10^{9} \mathrm{~m}^{3}$ and the effective adjusted water amount is $9.5 \times 10^{9} \mathrm{~m}^{3}$. The average annual water distribution and proportion of the provinces (cities) in WRA are shown in Table 1. 


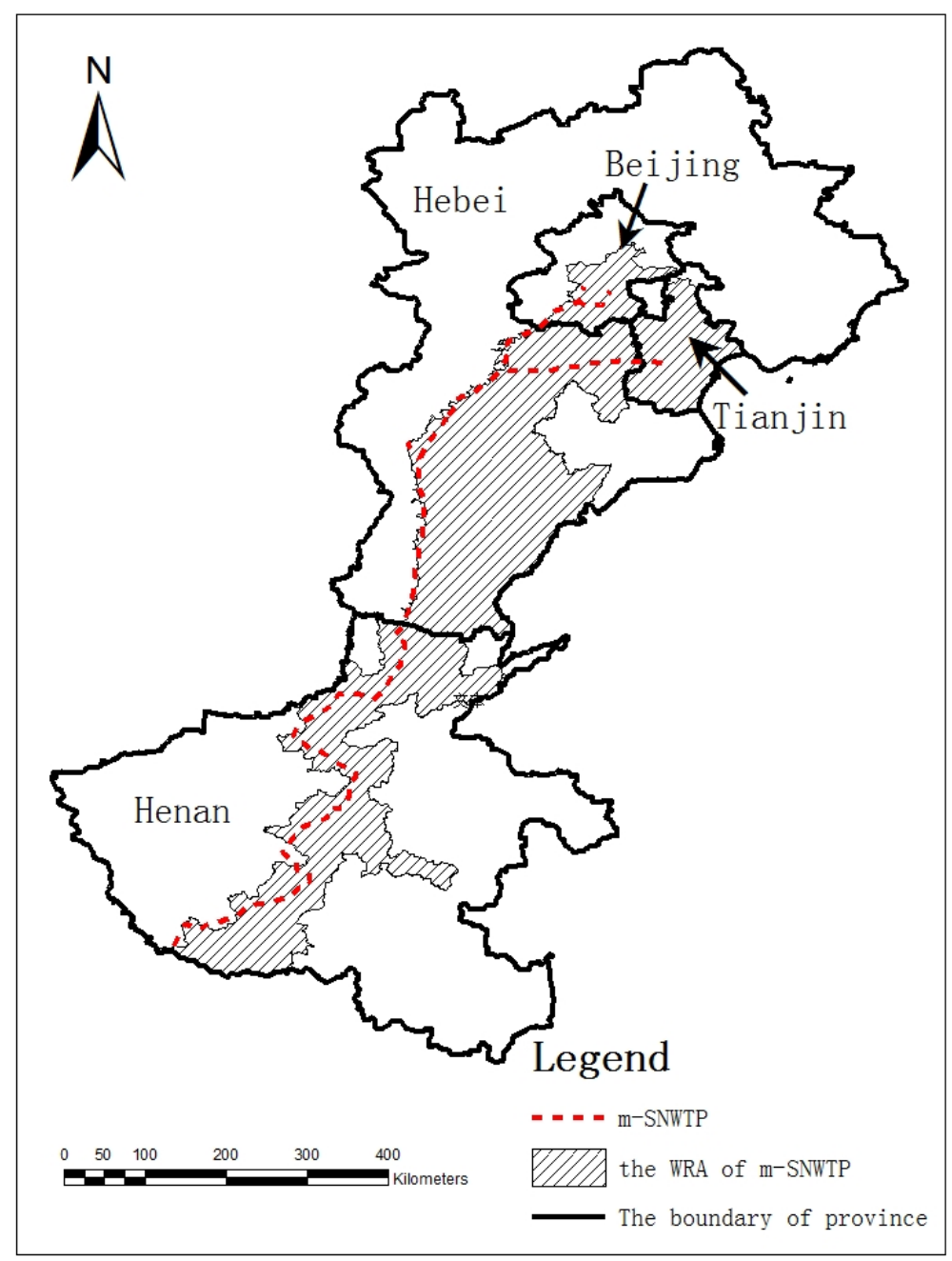

Figure 1. The location of the m-SNWTP

Table1. The average annual water distribution and proportion in the various WRA

\begin{tabular}{ccc}
\hline province (city) & Water account $/ 10^{8} \mathrm{~m}^{3}$ & Proportion $/ \%$ \\
\hline Henan & 37.7 & 39.7 \\
Hebei & 34.7 & 36.6 \\
Beijing & 12.4 & 13.0 \\
Tianjin & 10.2 & 10.7 \\
total & 95.0 & 100.0 \\
\hline
\end{tabular}

The first phase of the m-SNWTP has been running safely in the past three years. The actual water diversion volume of each WRA at water diverting outlets is showed in Figure 2. It can be seen that the dispatched water amount is increasing. Henan province consumes the most water, while Hebei province has the least amount of water consumed due to the progress of supporting projects. Up to the end of June 2018 , more than $1.4 \times 10^{10} \mathrm{~m}^{3}$ water had been supplied to the m-SNWTP benefited regions, including $8.65 \times 10^{8} \mathrm{~m}^{3}$ environmental water. The $\mathrm{m}$ SNWTP provides water source guarantee for the environment restoration. 


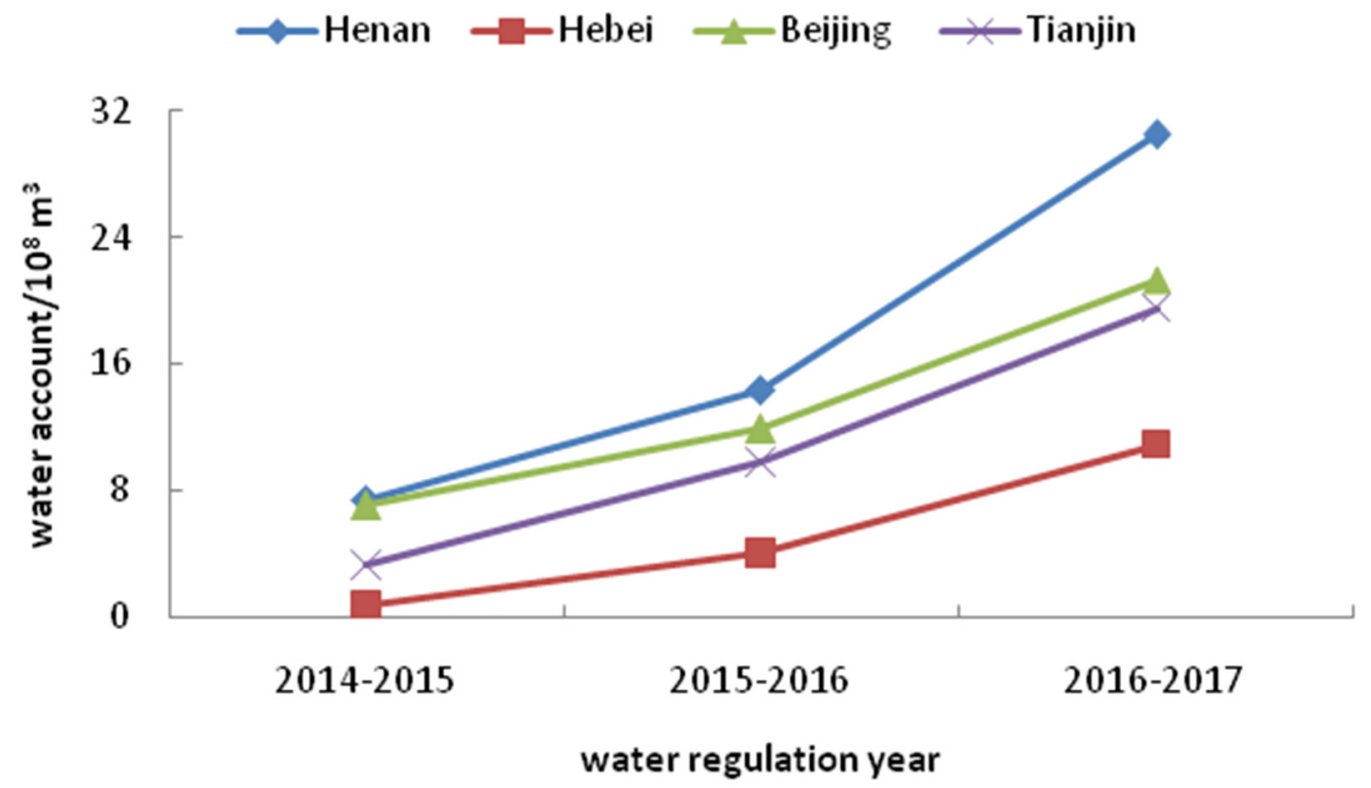

Figure 2. The water diversion volume of each WRA at water diverting outlets

\section{The groundwater protection in WRA}

The overexploitation of groundwater in the WRA is serious. The current overexploited amount is nearly $7.2 \times 10^{9} \mathrm{~m}^{3}$ in the WRA of $\mathrm{m}$-SNWTP, and the distribution of groundwater overexploitation is wide. The overexploitation has seriously damaged the ecological environment of the WRA, and has caused a series of ecological and environmental geological problems such as land subsidence, ground fissures, deterioration of water quality and salty water intrusion, which seriously restricts the sustainable development of economy and society ${ }^{[9-11]}$. The completion of the m-SNWTP has created favorable conditions for the groundwater overexploitation treatment in the WRA.

In 2013, the State Council approved the overall plan for reducing overexploited groundwater in the WRA of SNWTP, which requires reduce mining actively. The plan also clearly pointed out that the annual overexploited groundwater in the WRA will be reduced by $2.2 \times 10^{9} \mathrm{~m}^{3}$ compared with the base year by 2020 , and the balance of groundwater exploitation and recharge in urban areas will be basically realized. The annual groundwater overdraft in non-urban areas in the WRA will be reduced by $1.8 \times 10^{9}$ $\mathrm{m}^{3}$ compared with the base year by 2025 . After the $\mathrm{m}$ SNWTP operation, the cities in the WRA reduced the exploitation of groundwater by replacing water sources and adjusting planting structure. Consequently, the effect of groundwater recovery was remarkable. Figure 3 shows the situation of reduction and completion ratio of shortterm goal in various provinces and cities after the water diversion works.

$\square 2015 \square 2016 \square 2017$ aproportion

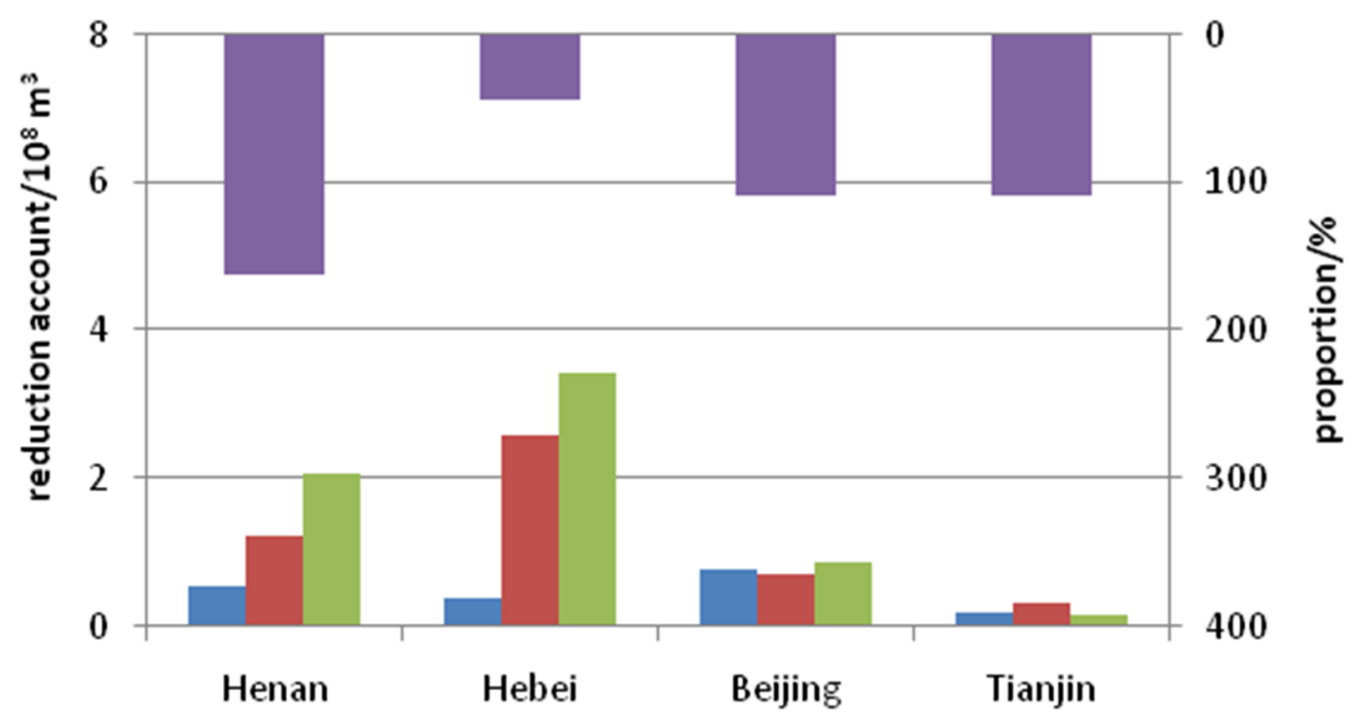

Figure 3. The reduction and completion ratio of short-term goal in the WRA 
It can be seen that the completion of the reduction goal and the situation of water consumption are generally consistent. With more water consumption, Henan, Beijing, and Tianjin have exceeded their reduction goals, while Hebei has not yet completed. However, the annual change shows that the reduction of Hebei Province is increasing year by year. In generally, over $1.326 \times 10^{9} \mathrm{~m}^{3}$ overexploited groundwater has been reduced in the urban areas of the WRA.

Comparing the results of groundwater monitoring in January 2018 and the same period of last year, it can be seen that the area of the shallow groundwater level rising, stable and declining in the WRA of SNWTP accounts for $16 \%, 50 \%$ and $34 \%$ of the WRA area respectively. The rising areas are mainly distributed in the plains south of the Yellow River in Henan Province, while the declining areas are mainly distributed in Shijiazhuang, Handan and Anyang. In general, the continuous decline of groundwater level has been effectively curbed.

\section{Conclusions}

The first phase of the m-SNWTP has been running safely in the past three years, and the accumulated water supply has exceeded $1.4 \times 10^{10} \mathrm{~m}^{3}$ water. There are 20 large and medium cities are benefited. The water supply volume in the WRA has been effectively improved, and the water quality of residents has been greatly improved. Besides, more than $1.326 \times 10^{9} \mathrm{~m}^{3}$ overexploited groundwater has been reduced in the urban areas of the WRA, and the continuous decline of groundwater level has been effectively curbed. Due to the increase in water supply, especially environmental water supply, the river and lake ecology has been effectively improved. However, the supporting projects in the WRA have not yet been fully completed so that the m-SNWTP has not reached the planned performance. In the future, it needs to be gradually optimized and improved the operation to ensure that the project benefits are fully utilized.

\section{Reference}

1. Xie Jia-ze, CHEN Zhi-kai. Water resources in China[J]. Acta Geographica Sinica, 1990(2):210-219.

2. Wang Yin-Tang, HU Si-Yi, Zhou Quan-Lin, et al. Optimal Water Operation for the Water Transfer Project from South to North (Middle Route)[J]. Advances in Water Science, 2001,12(1):72-80.

3. NIE Yan-hua, HUANG Guo-bing, CUI Xu, et al. Research on the target water level in emergency dispatch of the South-to-North Water Diversion Project[J]. South-to-North Water Transfers and Water Science \& Technology, 2017, 15(4): 198-202.

4. ZHONG Zhi-yu, LIU Guo-qiang, WU Ze-yu. Analysis and practices of water regulation in the Middle Route of South-to-North Water Transfer Project[J].South-to-North Water Transfers and Water Science \& Technology,2018,16(1):95-99,143.

5. Jiang Yu-Zhong, Zhao Hong-Li, Dong Yan-Jun, et al. Key Technology Research of Water Resources
Operation for Middle Route Project of South-toNorth Water Diversion[J]. South-to-North Water Transfers and Water Science \& Technology, 2007, $5(4): 1-5$.

6. WANG Hai-chao, JIANG Yun-zhong, LU Fan, et al. Inspiration of inter-basin water transfer abroad on operation of middle route of South-to-North Water Transfer Project[J]. Advances in Science and Technology of Water Resources, 2008, 28(2):79-83.

7. Wang Han-dong, Huang Shao-hua, Huang Hui-yong, et al. Design and realization of water quantity dispatching system of Middle Route Project of Southto-North Water Diversion[J]. Yangtze River, 2013, 44(9):100-103.

8. LI Yang, HU Gu-iquan, Yang Li-jin, et al. Preliminary Evaluation on Ecological Environmental Effect after Passing through Mid-route of South-to-North Water Diversion Project in Beijing[J]. Urban Geology, 2017,12(4):35-39.

9. ZHANG Guang-hui, FEI Yu-hong, LIU ke-yan,et al. Groundwater potential recovery and water level variation in the Shijiazhuang water-receiving area at the central line of the south-to-north water transfer project, China[J]. Geological Bulletin of China,2007,26(5):583-589.

10. Xiao Zhi-He, LI Tao. Effects of Tianjin Trunk Route of the Middle Route of South-to-North Water Diversion Project on Regional Groundwater Environment [J]. South-to-North Water Diversion and Water Science \& Technology, 2012, 10(1):6-10.

11. Zhao Rong, LI Zhen-Hai , Zhu Qiu-mei. Environmental Impact on Groundwater and Countermeasures of the Beijing Section of the Middle Route Project of the South-to-North Water Transfer[J]. South-to-North Water Transfers and Water Science \& Technology, 2010, 8(4):17-22. 